\title{
Key Abbreviations Used
}

\author{
ACA Agility Change Agent \\ ADT Agility Development Team \\ CEO Chief Executive Officer \\ EAfA Exploiting Agility for Advantage \\ LoB Line of Business \\ OD Organisation Development \\ TMT Top Management Team
}


